\title{
Assessment of carotid atherosclerotic disease using three-dimensional cardiovascular magnetic resonance vessel wall imaging: comparison with digital subtraction angiography
}

\author{
Zhenjia Wang ${ }^{1,2}$, Mi Lư ${ }^{3}$, Wen Liu', Tiejin Zheng ${ }^{4}$, Debiao Li ${ }^{5,6}$, Wei Yu ${ }^{1 *}$ (D) and Zhaoyang Fan ${ }^{5,6}$
}

\begin{abstract}
Background: A three-dimensional (3D) cardiovascular magnetic resonance (CMR) vessel wall imaging (VWI) technique based on 3D T1 weighted (T1w) Sampling Perfection with Application-optimized Contrast using different flip angle Evolutions (SPACE) has recently been used as a promising CMR imaging modality for evaluating extracranial and intra-cranial vessel walls. However, this technique is yet to be validated against the current diagnostic imaging standard. We therefore aimed to evaluate the diagnostic performance of 3D CMR VWI in characterizing carotid disease using intra-arterial digital subtraction angiography (DSA) as a reference.
\end{abstract}

Methods: Consecutive patients with at least unilateral $>50 \%$ carotid stenosis on ultrasound were scheduled to undergo interventional therapy were invited to participate. The following metrics were measured using 3D CMR WWI and DSA: lumen diameter of the common carotid artery (CCA) and segments $\mathrm{C} 1-\mathrm{C} 7$, stenosis diameter, reference diameter, lesion length, stenosis degree, and ulceration. We assessed the diagnostic sensitivity, specificity, accuracy, and receiver operating characteristic (ROC) curve of 3D CMR WWI, and used Cohen's kappa, the intraclass correlation coefficient (ICC), and Bland-Altman analyses to assess the diagnostic agreement between 3D CMR VWI and DSA.

Results: The ICC (all ICCs $\geq 0.96$ ) and Bland-Altman plots indicated excellent inter-reader agreement in all individual morphologic measurements by 3D CMR WWI. Excellent agreement in all individual morphologic measurements were also found between 3D CMR WWI and DSA. In addition, 3D CMR WWI had high sensitivity (98.4, 97.4, 80.0, 100.0\%), specificity $(100.0,94.5,99.1,98.0 \%)$, and Cohen's kappa $(0.99,0.89,0.84,0.96)$ for detecting stenosis $>50 \%$, stenosis $>70 \%$, ulceration, and total occlusion, respectively, using DSA as the standard. The AUC of 3D CMR WWI for predicting stenosis $>50$ and $>70 \%$ were 0.998 and 0.999 , respectively.

Conclusions: The 3D CMR WWI technique enables accurate diagnosis and luminal feature assessment of carotid artery atherosclerosis, suggesting that this imaging modality may be useful for routine imaging workups and provide comprehensive information for both the vessel wall and lumen.

Keywords: Vessel wall imaging, Carotid atherosclerotic disease, Magnetic resonance imaging, Validation

\footnotetext{
*Correspondence: yuweimd@163.com

'Department of Radiology, Beijing Anzhen Hospital, Capital Medical

University, No.2 Anzhen Road, Beijing 100029, China

Full list of author information is available at the end of the article
}

(c) The Author(s). 2020 Open Access This article is distributed under the terms of the Creative Commons Attribution 4.0 International License (http://creativecommons.org/licenses/by/4.0/), which permits unrestricted use, distribution, and reproduction in any medium, provided you give appropriate credit to the original author(s) and the source, provide a link to the Creative Commons license, and indicate if changes were made. The Creative Commons Public Domain Dedication waiver (http://creativecommons.org/publicdomain/zero/1.0/) applies to the data made available in this article, unless otherwise stated. 


\section{Background}

Carotid atherosclerosis is increasingly recognized as the leading cause of ischemic stroke [1-3]. Previous studies have shown that accurate evaluation of the degree of carotid stenosis would be beneficial for predicting the risk of stroke [4-6]. Digital subtraction angiography (DSA) is still considered the clinical gold standard for assessing the severity of carotid atherosclerosis [7, 8]. However, several major limitations of DSA have impeded its widespread use in routine imaging workups, including invasiveness and potential complications, radiation exposure, high costs, and difficulty in visualizing lesions with positive remodeling $[9,10]$. As a result, DSA is typically reserved for surgical interventions [7]. In contrast, ultrasound is a relatively cheap and portable method, and has been widely used for first-line diagnosis of atherosclerosis. However, ultrasound is susceptible to the level of experience of operators, and the use for patients with short, muscular necks is limited [11]. In contrast, high-resolution black-blood (BB) cardiovascular magnetic resonance (CMR) imaging can noninvasively visualize and quantify the vessel lumen and wall at a relatively low cost and without radiation exposure [12-16]. Hence, many groups have incorporated $\mathrm{BB} C \mathrm{CMR}$ in order to facilitate the diagnosis of carotid artery disease [17-21].

Recently, a three-dimensional (3D) CMR vessel wall imaging (VWI) technique based on 3D $\mathrm{T} 1$ weighted (T1w) Sampling Perfection with Application-optimized Contrast using different flip-angle Evolutions (SPACE) has become technically feasible and gained increasing attention for the diagnosis of atherosclerosis [22-26]. This technique is advantageous over conventional two-dimensional techniques, as it has sufficient BB effects, large spatial coverage, high isotropic resolution and signal-to-noise ratio (SNR), excellent scan efficiency, and remarkable cerebrospinal fluid (CSF) signal attenuation [23, 24, 27]. It therefore shows a unique capability for evaluating both lumen and wall features. Zhang et al. [27] demonstrated the reliability (excellent intra- and inter-observer agreement and scan-rescan reproducibility) of the $3 \mathrm{D}$ whole-brain intracranial VWI technique for quantifying intracranial vessel dimensions in 34 healthy subjects and 10 patients with known intracranial atherosclerotic disease. However, the diagnostic accuracy of 3D CMR VWI with respect to the luminal features involved in atherosclerosis has not been systematically validated against the clinical gold standard. Thus, we aimed to evaluate the performance of the 3D CMR VWI modality in diagnosing intra- and extra-cranial carotid atherosclerosis, using DSA as a reference.

\section{Methods}

This study protocol was approved by the local Institutional Review Board of Beijing Anzhen Hospital (Capital
Medical University, Beijing, China). Written informed consents were obtained from either the patients or their legal representatives prior to study entry.

\section{Participants}

Between July 2015 and June 2018, the study prospectively recruited consecutive patients. The patients had been scheduled for DSA and interventions due to presentation with amaurosis fugax, transient ischemic attack, or suspected recent ( $<14$ days) cerebrovascular ischemia and the diagnosis of $\geq 50 \%$ carotid artery stenosis by ultrasound. All enrolled patients underwent 3D CMR VWI within 7 days before DSA. Patients were excluded if they had metallic or pacemaker implants, severe claustrophobia, renal dysfunction, or if they were allergic to iodinated contrast material. Of note, patients who were unable to tolerate the $20 \mathrm{~min}$ CMR imaging protocol were also excluded.

\section{MR imaging protocol}

All examinations were performed on a 3-T whole-body CMR system (MAGNETOM Verio; Siemens AG Healthineers, Erlangen, Germany) using an 8-channel phased-array carotid surface coil (Shanghai Chenguang Medical technologies CO, LTD, Shanghai, China) and a 16-channel head coil (Siemens AG Healthineers). Patients were scanned in a supine, head-first position. After localization, 3D CMR VWI images were obtained using the following imaging parameters: sagittal orientation, echo time $(\mathrm{TE}) /$ repetition time $(\mathrm{TR})=14 / 900 \mathrm{~ms}$, field of view $(\mathrm{FOV})=180 \times 200 \times 120 \mathrm{~mm}^{3}, \quad$ spatial resolution $=0.63 \times 0.63 \times 0.63 \mathrm{~mm}^{3}$, matrix size $=284 \times$ $320 \times(208-240)$ with $7.7-25 \%$ partition oversampling, number of slices $=192$, number of averages $=1$, bandwidth $=488 \mathrm{~Hz} /$ pixel, parallel imaging (GRAPPA) factor $=2$, and scan time $=7 \mathrm{~min}$ and $3 \mathrm{~s}$. A trailing magnetization flip-down module was used to improve CSF signal attenuation [23]. The scan coverage included the C1-C7 segments of the bilateral internal carotid artery (ICA) and a distal portion of the common carotid artery (CCA).

\section{DSA imaging protocol}

DSA was performed with a digital angiography system (Innova 3100; General Electric Healthcare, Waukesha, Wisconsin, USA) operated by an experienced neurointerventionalist. Seldinger's technique was used to obtain patients' images of the internal carotid artery (ICA), including the anterior-posterior and lateral projections. A total of $10 \mathrm{~mL}$ (rate, $4-5 \mathrm{~mL} / \mathrm{sec}$ ) of contrast medium (Iopamidol 370, Bracco, Shanghai, China) was injected into the ICA. The parameters were as follows: matrix size $=1024 \times 1024$, spatial resolution $=2.75 \mathrm{LP} / \mathrm{mm}$, and $\mathrm{FOV}=30 \mathrm{~cm}$. 


\section{Image review}

One neurointerventionalist with 17 years of experience (T.Z.) reviewed the DSA images and performed morphologic quantification at a workstation (AW4.7; General Electric Healthcare). The 3D CMR VWI images was reviewed and measured on a workstation (Leonardo, Siemens Healthineers, Erlangen, Germany) equipped with the multiplanar reconstruction (MPR) functionality.

Image quality (IQ) of CMR VWI was first assessed by an experienced reviewer (Y.W.) using a 4-point scale, where 1 = poor (insufficient for diagnosis, low SNR and obscured vessel wall or lumen boundaries), 2 =marginal (barely diagnosable, moderate SNR with a few motion or blood artifacts, distinguishable vessel wall and lumen boundary), 3 =good (appropriate for diagnosis, good SNR, distinguishable vessel wall, but partially obscured vessel lumen and wall boundaries), and $4=$ excellent (sufficient for diagnosis; high SNR without artifacts, displaying vessel lumen boundary and wall clearly).
Examples of IQ at different scale levels are provided in Online Figure 1. Images with IQ of 1 were excluded from further analyses [28, 29].

Each ICA was divided into 7 segments (C1-C7) using the Bouthillier classification [30]. Data corresponding to each segment was measured in the 0.63mm-thick cross-sections reformatted from 3D CMR VWI. A case example is provided in Fig. 1. The following variables were measured from the $3 \mathrm{D} C M R$ VWI and corresponding DSA images: 1) lumen diameter (i.e. the lumen diameter of the normal CCA and each ICA segment, stenosis diameter, and reference diameter); 2) stenosis degree (\%), calculated according to the North American Symptomatic Carotid Endarterectomy Trial criteria [31]; 3) lesion length, measured as the maximum range of the lesion at the sagittal plane); and 4) presence of ulceration, defined as carotid plaque surface with fissuring or fibrous cap rupture. If a carotid artery had

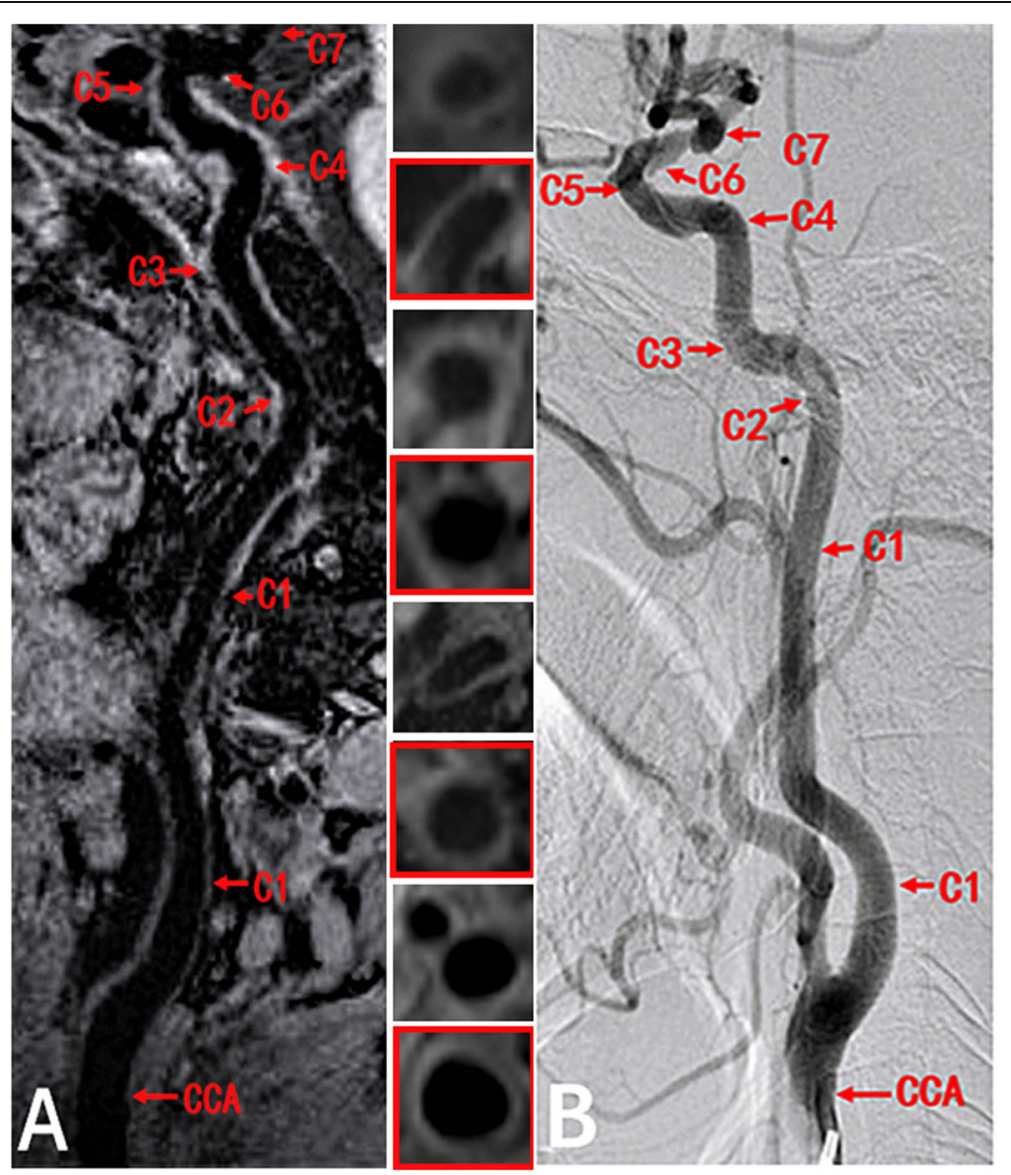

Fig. $13 D$ CMR WWI and DSA images of CCA. Both a, a sagittal 3D cardiovascular magnetic resonance (CMR) vessel wall imaging (VWI) image, and b, a lateral projection of a digital subtraction angiography (DSA) image, depict the lumen diameters of the common carotid artery (CCA) and C1C7 segments in a 59-year-old man. The cross-sectional views at 8 locations are shown with numbered sub-windows, including CCA and internal carotid artery (ICA) (C1-C7). CCA, common carotid artery; 3D CMR WWI, three-dimensional cardiovascular magnetic resonance vessel wall imaging; DSA, digital subtraction angiography; ICA, internal carotid artery 
multiple stenoses, the narrowest one was evaluated. The severity of stenosis was subsequently classified according to the following criteria: no stenosis $=0 \%$, mild stenosis $=0-29 \%$, moderate stenosis $=30-69 \%$, severe stenosis $=70-99 \%$, and total occlusion $=100 \%$. Two experienced radiologists (Z.W. and L.W.) blinded to the clinical information and DSA results, performed the above quantitatively analyses on the 3D CMR VWI images.

\section{Statistical analysis}

Continuous variables are presented as mean \pm standard deviation (SD), and categorical variables are reported as frequencies (percentages), as appropriate. Kolmogorov-Smirnov tests were used to access whether the measurements were normally distributed. Paired $t$-tests were used to test for the significant differences between readers or methods. The sensitivity, specificity, accuracy, positive predictive value, negative predictive value, and Cohen's kappa with 95\% confidence intervals of the 3D CMR VWI images were calculated for diagnosing total occlusion, carotid artery stenosis, and ulceration using DSA images as a reference. For continuous variables, the agreement between 3D CMR VWI and DSA, as well as the agreement between two CMR readers were assessed by using the intraclass correlation coefficient (ICC) and Bland-Altman analyses. The level of the agreement was categorized as follows: poor $(\mathrm{ICC}=0-0.20)$; fair $\quad(\mathrm{ICC}=0.21-0.40)$; moderate ( $\mathrm{ICC}=0.41-0.60)$; good $(\mathrm{ICC}=0.61-0.80)$; and excellent (ICC $=0.81-1.00)$ [32]. Receiver operating characteristic (ROC) curves were used to evaluate the performance of 3D CMR VWI at the DSA-stenosis cutoff values of 50 and $70 \%$, respectively. All statistical analyses were performed using
SPSS software, version 19.0 (Statistical Package for the Social Sciences, International Business Machines, Inc., Armonk, New York, USA). P-values $<0.05$ were considered to indicate statistical significance.

\section{Results}

Image quality

A total of 67 patients successfully underwent 3D CMR VWI and DSA. The overall image quality for 3D CMR VWI was $3.13 \pm 0.73$. Two patients were excluded from the study due to severe blood flow artifacts at the carotid bifurcation $(n=1)$ and severe motion artifacts $(\mathrm{n}=1)$. In total, 65 patients were included in the final analyses. A detailed flow chart of the study design is presented in Fig. 2.

\section{Patient characteristics}

Baseline demographics, medical history, and biochemical characteristics of the study population are shown in Table 1. The mean age of patients was $63.6 \pm 5.5$ years, and $52(80.0 \%)$ subjects were male.

\section{Inter-reader agreement of the 3D CMR VWI images}

Of the 130 arteries, 31 total occlusions were identified by two CMR image readers with $100 \%$ interreader agreement. A case example of the total occlusion depicted by $3 \mathrm{D}$ CMR VWI is shown in Fig. 3a. The results of the morphologic measurements among the remaining 99 arteries and the arteries with stenosis $>50 \%$ identified by ultrasonography are summarized in Table 2. All ICCs of all morphologic indices were $\geq 0.96$, indicating excellent inter-reader reproducibility. But there are significant differences among some metrics between CMR reader 1 and reader 2 . The Bland-Altman plots of

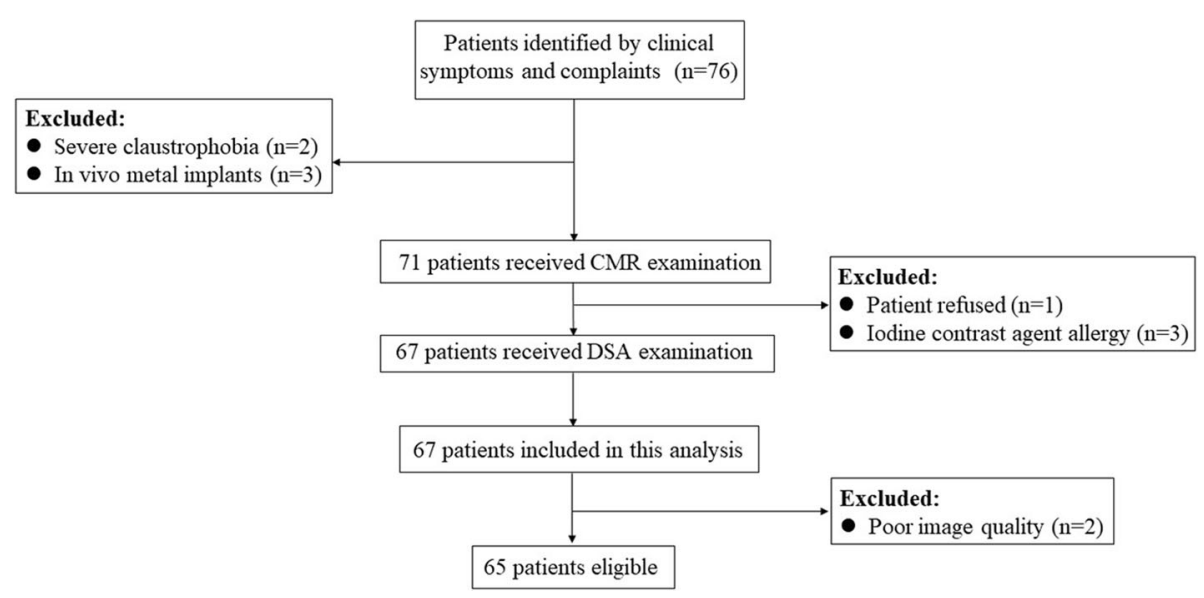

Fig. 2 Flow chart of the study design. DSA, digital subtraction angiography; CMR, cardiovascular magnetic resonance 
Table 1 Characteristics of the study's cohort

\begin{tabular}{|c|c|}
\hline Variables & Participants \\
\hline Age, years & $63.6 \pm 8.56$ \\
\hline Males, $n(\%)$ & $52(80.0 \%)$ \\
\hline Hypertension, n (\%) & $44(67.7 \%)$ \\
\hline Hyperlipidemia, $n$ (\%) & $55(84.6 \%)$ \\
\hline Diabetes mellitus, $n$ (\%) & $35(53.9 \%)$ \\
\hline Coronary artery disease, $n(\%)$ & $51(78.5 \%)$ \\
\hline Current smokers, $n(\%)$ & $47(72.3 \%)$ \\
\hline Total cholesterol, $\mathrm{mmol} \cdot \mathrm{L}^{-1}$ & $3.76 \pm 1.26$ \\
\hline $\mathrm{HDL}$ cholesterol, $\mathrm{mmol} \cdot \mathrm{L}^{-1}$ & $1.03 \pm 0.39$ \\
\hline $\mathrm{LDL}$ cholesterol, $\mathrm{mmol} \cdot \mathrm{L}^{-1}$ & $2.24 \pm 0.98$ \\
\hline Triglyceride, $\mathrm{mmol} \cdot \mathrm{L}^{-1}$ & $1.36 \pm 0.70$ \\
\hline Homocysteine, umol.L-1 & $14.38 \pm 9.14$ \\
\hline Glucose, mmol.L-1 & $6.77 \pm 2.02$ \\
\hline$L P(a), g \cdot L-1$ & $0.24 \pm 0.28$ \\
\hline \multicolumn{2}{|l|}{ MTHFR677C-T } \\
\hline$C C, n(\%)$ & $6(9.2 \%)$ \\
\hline$C T, n(\%)$ & $40(61.5 \%)$ \\
\hline$\pi, n(\%)$ & $19(29.3 \%)$ \\
\hline
\end{tabular}

Data are presented as $n(\%)$ or mean \pm standard deviation. HDL, high density lipoprotein, LDL, low density lipoprotein, LP(a), lipoprotein (a), MTHFR677C-T, methylenetetrahydrofolate reductase $677 \mathrm{C}$ and $\mathrm{T}$

the stenosis degree and CCA diameter between CMR reader 1 and reader 2 in all of the arteries without total occlusion are shown in Fig. 4. The mean differences were $0.3 \%$ and $-0.07 \mathrm{~mm}$, respectively.

\section{Agreement between 3D CMR VWI and DSA}

Of the 130 arteries, 29 total occlusions were identified by DSA. 3D CMR VWI revealed all the 29 total occlusions but mistook two pseudo-occlusive vessels for total occlusion. Thus, the sensitivity, specificity, accuracy, positive predictive value (PPV), negative predictive value (NPV), and Cohen's kappa of 3D CMR VWI for diagnosing total occlusion were 100.0, 98.0, 98.5, 93.5, 100.0\% and 0.96, respectively. A case example of the total occlusion assessed by DSA is shown in Fig. 3b. The agreements in the quantitative measurements between 3D CMR VWI reader 1 and DSA for the remaining 101 arteries and 50 arteries with stenosis $>50 \%$ identified by ultrasonography are summarized in Table 3. The ICC values indicated excellent agreement for all of the morphologic measurements between 3D CMR VWI and DSA (all ICCs $\geq 0.90)$ in the remaining 101 arteries. In the arteries with stenosis $>50 \%$ detected by ultrasonography, there was excellent agreement for all metrics (all ICCs $\geq 0.84$ ) between 3D CMR VWI imaging and DSA. The Bland-Altman plots of the stenosis degree

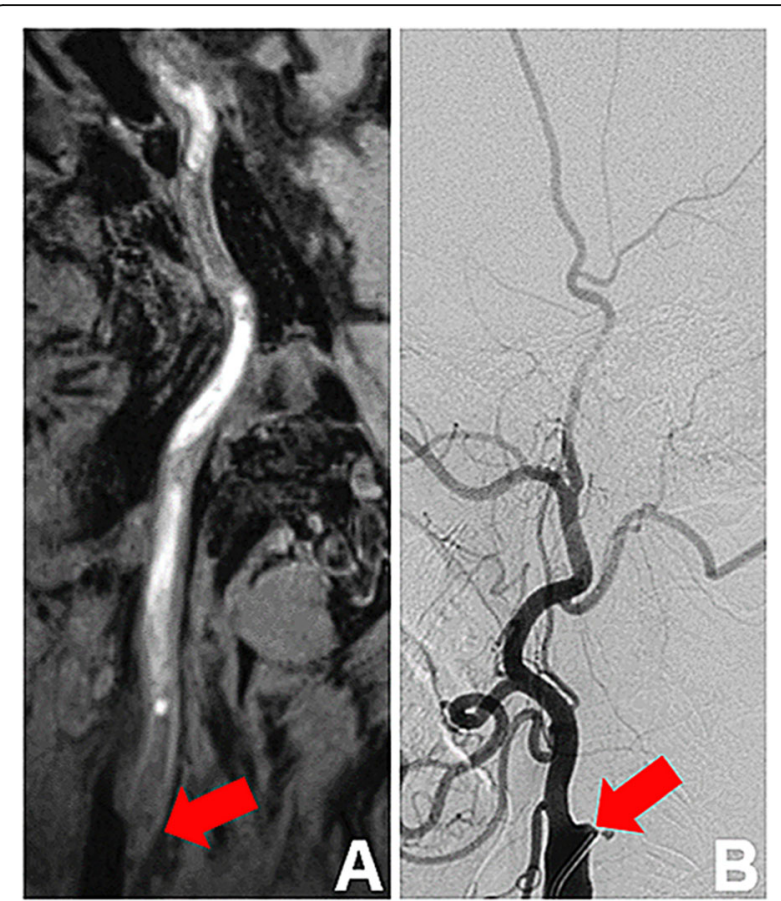

Fig. 3 3D CMR WWI and DSA images of CCA of total occlusion. Both $\mathbf{a}$, a sagittal 3D CMR WWI image, and $\mathbf{b}$, a lateral projection of a DSA image depict total occlusion of the internal carotid artery (arrow) in a 67- year-old man. 3D CMR WWI, three-dimensional cardiovascular magnetic resonance vessel wall imaging; DSA, digital subtraction angiography

and CCA diameter between CMR and DSA in all arteries without total occlusion are presented in Fig. 5. The mean differences were $1.9 \%$ and $-1.98 \mathrm{~mm}$, respectively.

\section{Diagnostic accuracy of 3D CMR VWI imaging}

Using DSA as the reference, the sensitivity, specificity, positive predictive value, negative predictive value, accuracy, and Cohen's kappa of 3D CMR VWI for detecting stenosis $>50 \%$, stenosis $>70 \%$, and total occlusion are presented in Table 4. Both CMR readers were able to identify the lesions. Of the 130 arteries, 20 (12.3\%) arteries had ulcerations according to DSA, whereas CMR reader 1 detected 16 (80\%) ulcerations on 3D CMR VWI. The sensitivity, specificity and Cohen's kappa values were 80\% (95\% confidence interval [CI]: 62.5, 97.5\%), 99.1\% (95\% CI: $97.3,100 \%)$ and 0.84 (95\% CI: $0.67,1.00)$, respectively. In addition, 3D CMR VWI also had a high level of sensitivity (98.4, 97.4, 100.0\%), specificity (100.0, 94.5, 98.0\%), and Cohen's kappa (0.99, 0.89, 0.96 ) for detecting stenosis $>50 \%$, stenosis $>70 \%$, and total occlusion, respectively, by MR reader 1 . Figure 6 shows the ROC curve reflecting the diagnostic capability of 3D CMR VWI in the entire 


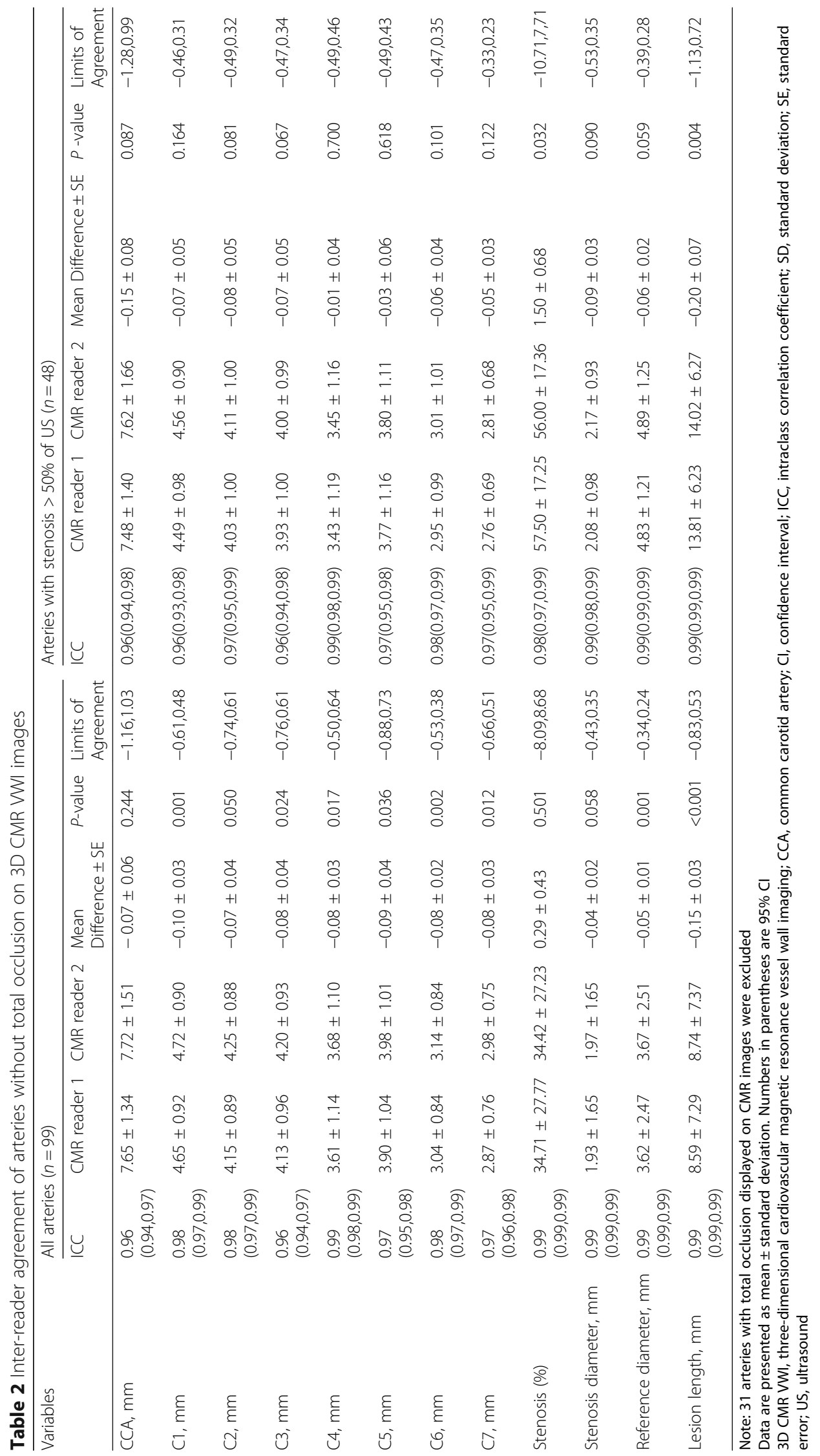



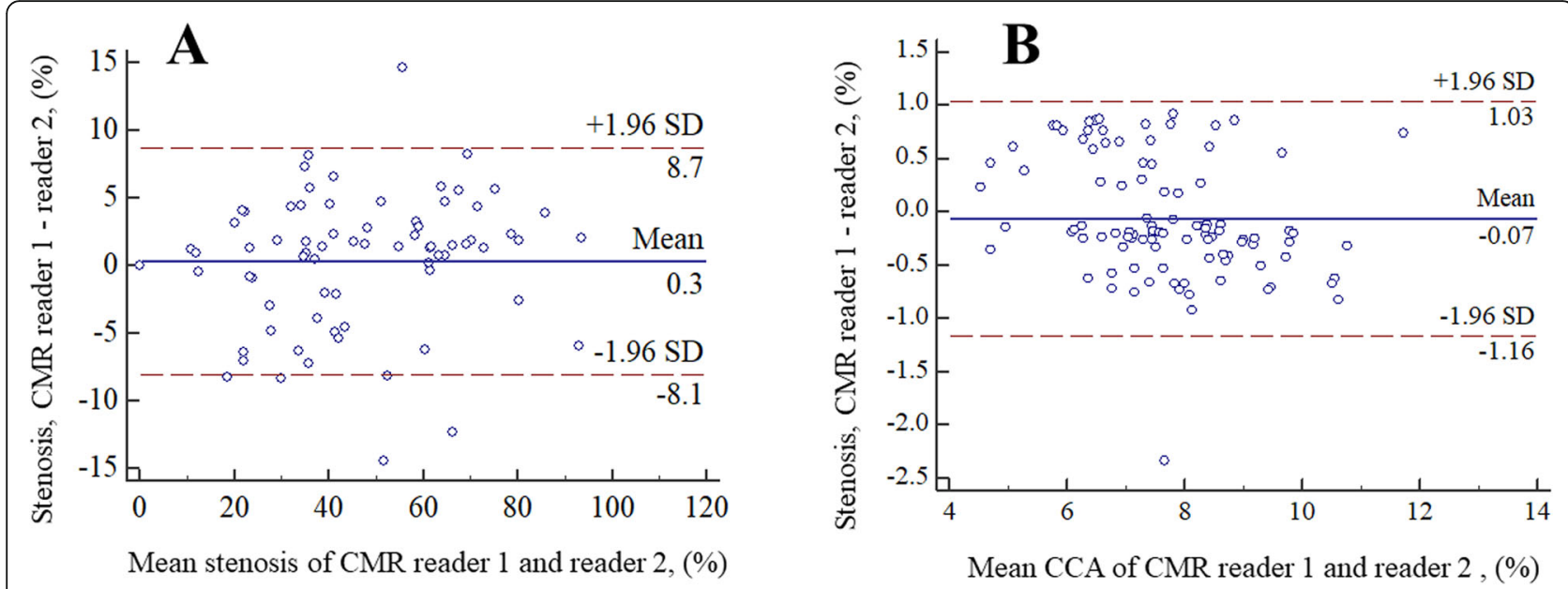

Fig. 4 Bland-Altman plots of morphologic measurements between CMR reader 1 and reader 2 in all arteries without total occlusion. The solid lines represent the mean difference, and the dashed lines indicate the $95 \%$ limits of agreement. CCA, common carotid artery; SD, standard deviation; CMR, cardiovascular magnetic resonance

cohort when the threshold of stenosis was set at 50 and $70 \%$, respectively.

\section{Discussion}

This study confirms the accuracy of the 3D CMR VWI technique based on the 3D T1w SPACE sequence for the diagnosis of carotid atherosclerosis, particularly the assessment of various luminal features, in symptomatic patients with $\geq 50 \%$ carotid artery stenosis. We found that the 3D CMR VWI technique is highly consistent with DSA when measuring the lumen diameter of the CCA and segments C1-C7 segments, as well as the stenosis diameter, stenosis degree, and reference diameter. Additionally, this technique showed excellent accuracy for diagnosing total occlusion, ulcerative lesions, and moderate to severe stenosis. Hence, our results justify the increasing use of 3D CMR VWI as a reliable, accurate, and non-invasive imaging modality for diagnosing carotid artery disease.

Analysis of inter-observer reproducibility is an essential requirement for validation of an imaging modality. Zhang et al. [27] previously performed a comprehensive reliability analysis for the 3D wholebrain CMR VWI technique. However, this study was limited by the small sample size of clinical patients. In our study, two experienced radiologists evaluated the intra- and extra-cranial carotid walls using 3D CMR VWI in 65 patients. Our results showed excellent inter-observer reproducibility for measurement of the lumen diameter of the CCA and segments C1-C7 of the carotid artery, stenosis diameter, stenosis degree, and reference diameter. There were systematic differences in diameter measurements, particularly at large-size vessel segments, between reader 1 and 2 . This may be due to the different measurement habits among readers and non-circular lumen shapes of the large-size segments. Nevertheless, these differences were all submillimeters and not clinically relevant for diagnosis. Thus, 3D CMR VWI is considered to be a reliable imaging modality for the quantification of luminal morphologic features.

In this study, we evaluated the agreement between 3D CMR VWI and DSA. The morphologic measurements, including the lumen diameter of the CCA and segments $\mathrm{C} 1-\mathrm{C} 7$, and the stenosis diameter, stenosis degree, reference diameter, and lesion length, as measured by 3D CMR VWI, displayed excellent accordance with those measured by DSA (ICC > 0.84). One possible reason for the excellent performance of the 3D CMR VWI technique is that a non-selective excitation radio-frequency pulse has been used to shorten the echo time, which helps improve the SNR of images [23, 24, 27]. Additionally, 3D CMR VWI provided a high isotropic resolution of $0.63 \times 0.63 \times$ $0.63 \mathrm{~mm}^{3}$, and achieves good flexibility with regard to image visualization, which thereby enables the visualization of plaques located in the tortuous and deep-seated intracranial carotid. Furthermore, dedicated CSF signal suppression is implemented in this technique, which can help to visualize the outer boundaries of the vessel wall $[23,27]$.

However, our study showed some systematic differences between CMR VWI and DSA. Firstly, the lumen diameter tended to be underestimated by VWI at larger sized vessel segments. This discrepancy might be caused by the underlying differences in 


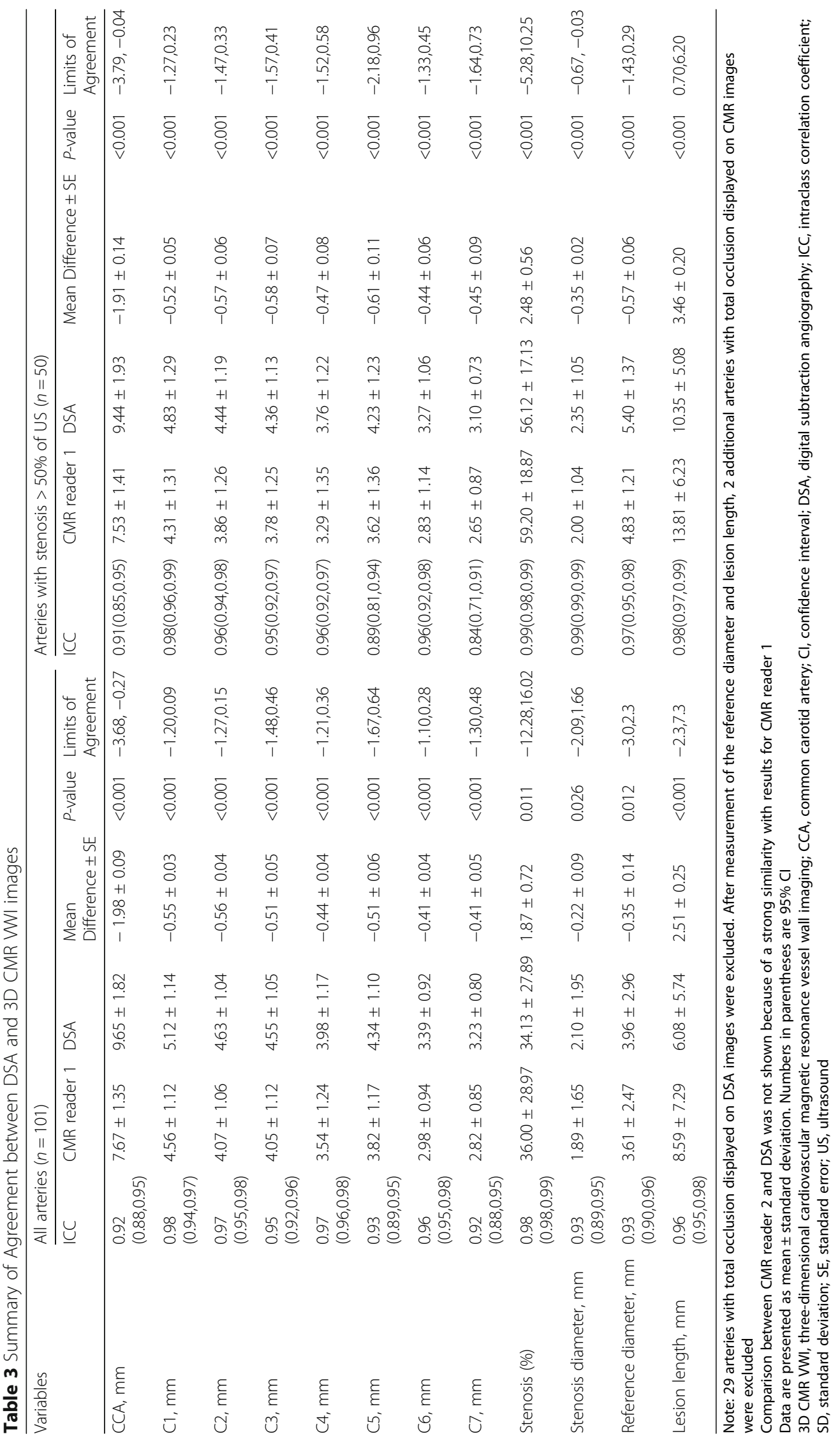



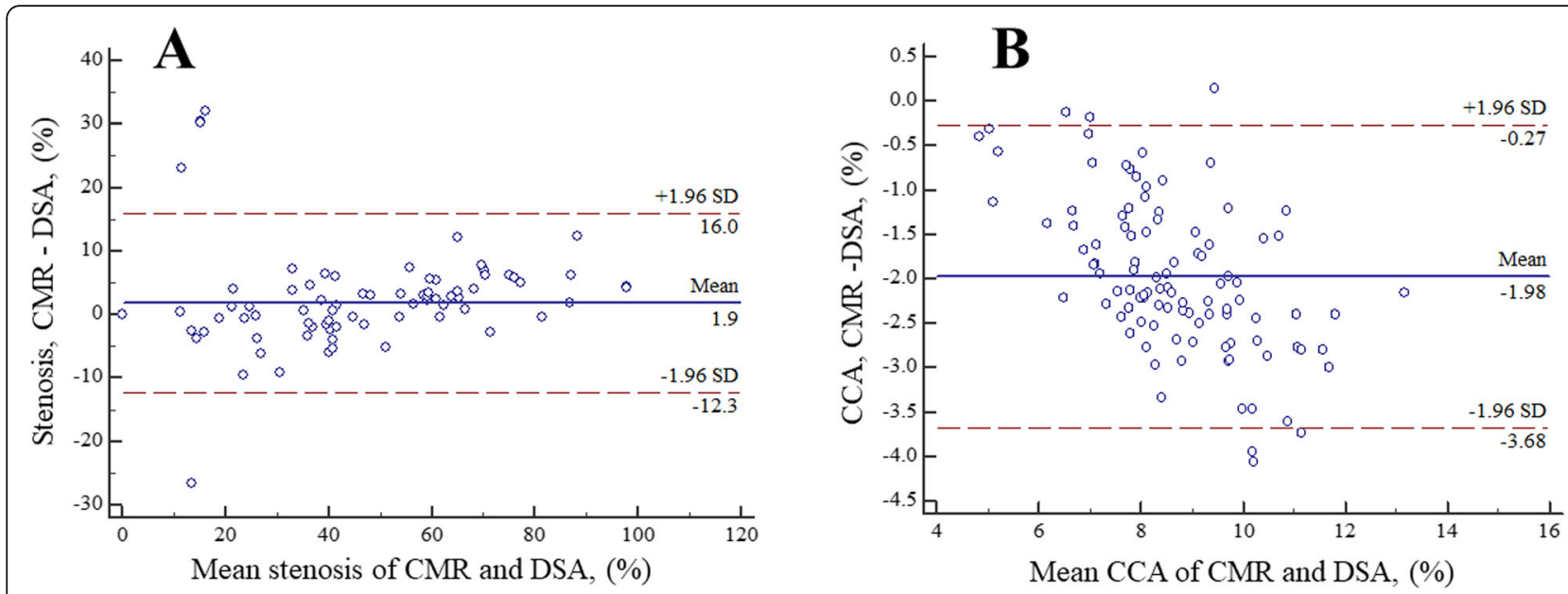

Fig. 5 Bland-Altman plots of morphologic measurements as measured by CMR and DSA in all arteries without total occlusion. The solid lines represent the mean difference, and the dashed lines indicate the 95\% limits of agreement. CCA, common carotid artery; DSA, digital subtraction angiography; CMR, cardiovascular magnetic resonance; SD, standard deviation

image acquisition and measurement. Specifically, DSA is a projection-based imaging modality and the distance between two parallel vessel boundaries from the imaging perspective is inherently the largest possible diameter of the vessel from that perspective. However, diameter measurement on VWI is performed from reformatted cross-sectional images and derived by averaging two perpendicular hand-drawn straight-line distances. Either of these lines is necessarily aligned with the DSA perspective, and more importantly, each distance may not represent the true diameter at that angle if the straight line misses the lumen center, thus underestimating the diameter. The averaging procedure would further make the measured diameter smaller, particularly at larger vessel segment that are not necessarily round. Secondly, the lesion lengths measured by 3D CMR VWI are about $3 \mathrm{~mm}$ longer than those measured by DSA in arteries with $>50 \%$ stenosis. The potential reason may be the distal portion of a wedge-shaped plaque being regarded as the reference on DSA [33, 34].

In our study, 3D CMR VWI showed a poor ability to identify pseudo-occlusion and small ulcerations (width $<1.5 \mathrm{~mm}$ ), compared with DSA. Mistaking pseudo-occlusion for total occlusion on 3D CMR VWI might be explained by the fact that DSA has much better spatial and temporal resolution than $3 \mathrm{D}$ CMR VWI. This is in line with the findings of a previous study [20]. Missing small ulceration is attributed not only to the poorer spatial resolution but also to the existence of tiny calcification in the surface of the lumen $[12,20]$. Therefore, we should

Table 4 Sensitivity, Specificity, PPV, NPV, Accuracy and Cohen's kappa of 3D CMR WWI images analysis using DSA as reference

\begin{tabular}{|c|c|c|c|c|c|c|c|c|c|c|c|c|}
\hline \multirow[t]{2}{*}{ Variables } & \multicolumn{2}{|l|}{ Sensitivity } & \multicolumn{2}{|l|}{ Specificity } & \multicolumn{2}{|l|}{ PPV } & \multicolumn{2}{|l|}{ NPV } & \multicolumn{2}{|l|}{ Accuracy } & \multicolumn{2}{|c|}{$\begin{array}{l}\text { Cohen's } \\
\text { kappa }\end{array}$} \\
\hline & CMR1 & CMR2 & CMR1 & CMR2 & CMR1 & CMR2 & CMR1 & CMR2 & CMR1 & CMR2 & CMR1 & CMR2 \\
\hline $\begin{array}{l}\text { Total } \\
\text { Occlusion } \\
(n=29)\end{array}$ & $\begin{array}{l}100.0 \\
(100.0 \\
100.0)\end{array}$ & $\begin{array}{l}100.0 \\
(100.0 \\
100.0)\end{array}$ & $\begin{array}{l}98.0 \\
(95.3 \\
100.0)\end{array}$ & $\begin{array}{l}98.0(95.3 \\
100.0)\end{array}$ & $\begin{array}{l}93.5 \\
(84.9 \\
100.0)\end{array}$ & $\begin{array}{l}93.5(84.9, \\
100.0)\end{array}$ & $\begin{array}{l}100.0 \\
(100.0 \\
100.0)\end{array}$ & $\begin{array}{l}100.0 \\
(100.0 \\
100.0)\end{array}$ & $\begin{array}{l}98.5 \\
(96.3,100.0)\end{array}$ & $\begin{array}{l}98.5 \\
(96.3,100.0)\end{array}$ & $\begin{array}{l}0.96 \\
(0.79 \\
1.00)\end{array}$ & $\begin{array}{l}0.96 \\
(0.79, \\
1.00)\end{array}$ \\
\hline $\begin{array}{l}\text { Ulcer } \\
(n=20)\end{array}$ & $\begin{array}{l}80.0 \\
(62.5,97.5)\end{array}$ & $\begin{array}{l}85.0(69.4 \\
100.0)\end{array}$ & $\begin{array}{l}99.1 \\
(97.3 \\
100.0)\end{array}$ & $\begin{array}{l}98.2 \\
(95.7,100.0)\end{array}$ & $\begin{array}{l}94.1 \\
(82.9,100)\end{array}$ & $\begin{array}{l}89.5 \\
(75.7,100.0)\end{array}$ & $\begin{array}{l}96.4 \\
(93.1,99.9)\end{array}$ & $\begin{array}{l}97.3 \\
(94.3,100.0)\end{array}$ & $\begin{array}{l}96.2 \\
(92.8,99.5)\end{array}$ & $\begin{array}{l}96.2(92.8, \\
99.5)\end{array}$ & $\begin{array}{l}0.84 \\
(0.67 \\
1.00)\end{array}$ & $\begin{array}{l}0.85 \\
(0.68 \\
1.00)\end{array}$ \\
\hline \multicolumn{13}{|l|}{ Stenosis (\%) } \\
\hline $\begin{array}{l}>50 \% \\
(n=60)\end{array}$ & $\begin{array}{l}98.4 \\
(95.2,100.0)\end{array}$ & $\begin{array}{l}96.8 \\
(92.4,100.0)\end{array}$ & $\begin{array}{l}100.0 \\
(100.0 \\
100.0)\end{array}$ & $\begin{array}{l}98.5(95.7 \\
100.0)\end{array}$ & $\begin{array}{l}100.0 \\
(100.0 \\
100.0)\end{array}$ & $\begin{array}{l}98.4(95.2, \\
100.0)\end{array}$ & $\begin{array}{l}98.6 \\
(95.8 \\
100.0)\end{array}$ & $\begin{array}{l}97.1 \text { (93.1, } \\
100.0)\end{array}$ & $\begin{array}{l}99.2(97.7 \\
100.0)\end{array}$ & $\begin{array}{l}97.7 \text { (95.1, } \\
100.0)\end{array}$ & $\begin{array}{l}0.99 \\
(0.81 \\
1.00)\end{array}$ & $\begin{array}{l}0.95 \\
(0.78, \\
1.00)\end{array}$ \\
\hline $\begin{array}{l}>70 \% \\
(n=38)\end{array}$ & $\begin{array}{l}97.4(92.5 \\
100.0)\end{array}$ & $\begin{array}{l}97.4(92.5 \\
100.0)\end{array}$ & $\begin{array}{l}94.5 \\
(89.8,99.2)\end{array}$ & $\begin{array}{l}97.8 \\
(94.8,100.0)\end{array}$ & $\begin{array}{l}88.4 \\
(78.8 \\
98.0)\end{array}$ & $\begin{array}{l}95.0 \\
(88.2,100.0)\end{array}$ & $\begin{array}{l}98.9 \\
(96.6 \\
100.0)\end{array}$ & $\begin{array}{l}98.9 \text { (96.7, } \\
100.0)\end{array}$ & $\begin{array}{l}95.4 \\
(91.8,99.0)\end{array}$ & $\begin{array}{l}97.7 \\
(95.1,100.0)\end{array}$ & $\begin{array}{l}0.89 \\
(0.72 \\
1.00)\end{array}$ & $\begin{array}{l}0.95 \\
(0.77, \\
1.00)\end{array}$ \\
\hline
\end{tabular}

All data, except for Cohen's kappa, are percentages. Numbers in parentheses are $95 \% \mathrm{Cl}$

3D CMR VWI, cardiovascular magnetic resonance three-dimensional vessel wall imaging; DSA, digital subtraction angiography; NPV, negative predictive value; PPV, positive predictive value 

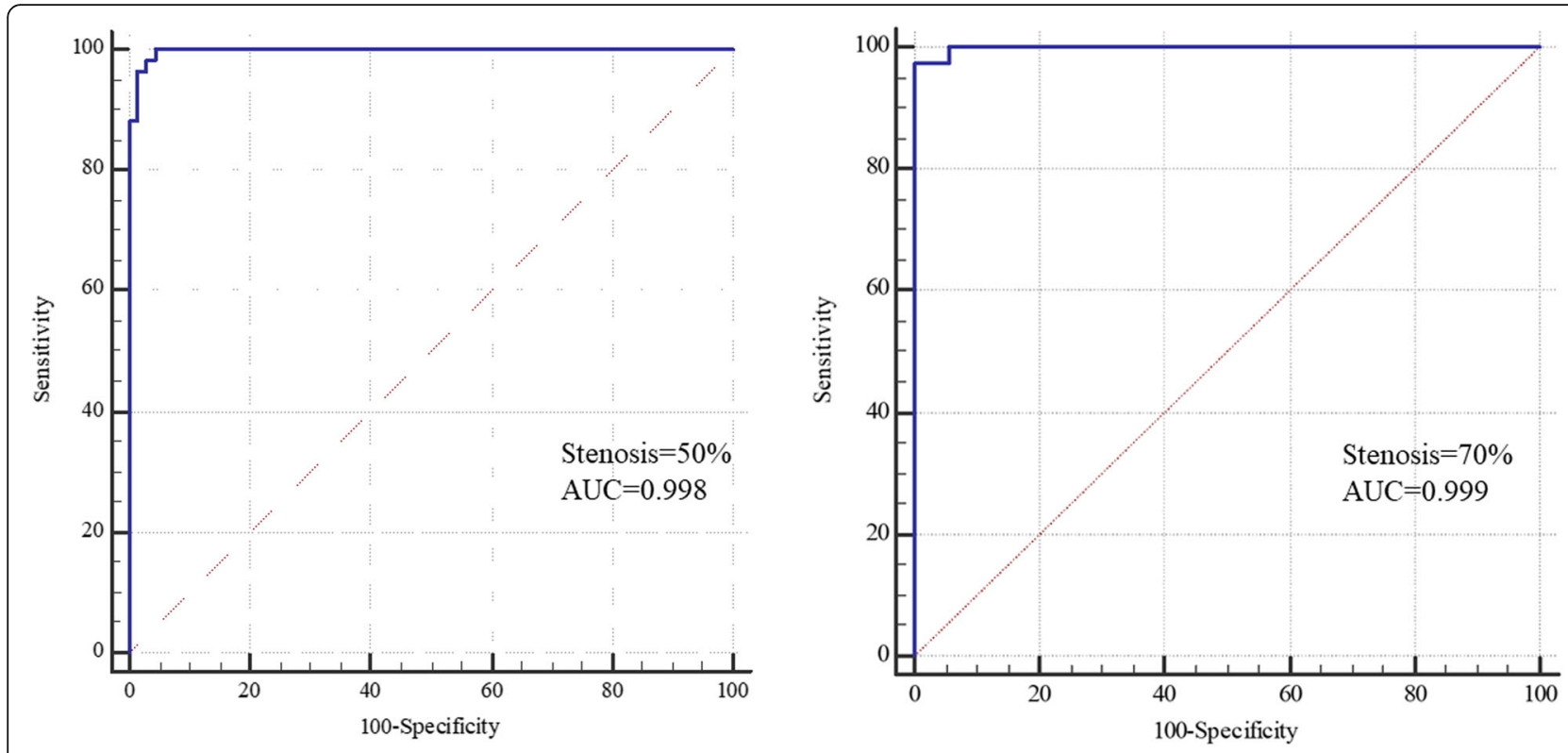

Fig. 6 ROC curves of 3D CMR VWI according to various stenosis degree cutoff values. 3D CMR WWI, three-dimensional cardiovascular magnetic resonance vessel wall imaging; AUC: area under the curve; ROC: receiver operating characteristic

pay more attention to the irregular calcification on the 3D CMR VWI images so as to detect ulceration properly. A previous study showed that bright-blood sequences are highly accurate in the detection of ulcerations because of a good depiction of the luminal boundary. Therefore, future studies which examine the combination of bright-blood sequences with 3D T1w SPACE sequences for the comprehensive diagnosis of ulceration are warranted [35].

Zhao et al. [20] performed a study similar to the current one, and showed that $3 \mathrm{D}$ BB MR imaging could accurately diagnose patients with moderate to severe carotid artery stenosis, which is consistent with the findings of our study. One potential limitation of their study is that they only assessed the consistency and accuracy of 3D BB MR in carotid lesions with $>50 \%$ stenosis. However, an increasing number of studies have found that carotid lesions with low-grade stenosis may also lead to stroke [4-6]. Moreover, cerebral angiography is often required before carotid artery stent implantation in order to observe the intracranial Willis ring opening and cerebral blood flow perfusion. Therefore, our study performed a comprehensive analysis of the consistency and accuracy of 3D CMR VWI in lesions with both less than and greater than 50\% stenosis. We showed excellent consistency for measurement of lumen diameter and stenosis in all arteries between 3D CMR VWI and DSA. However, CMR VWI may be less accurate for more severe lesions. Briefly, there are two potential reasons why this may be the case. First, 3D CMR VWI has poorer spatial resolution than DSA, meaning that the measurement errors induced by partial volume effect become more evident in cases of severe stenoses. Second, blood signal suppression may become more challenging as the severity of stenosis increases, resulting in residual flow artifacts particularly at juxta-luminal locations. Another limitation of their study was that they only assessed lesions located at the level of carotid bifurcation and not segments $\mathrm{C} 2-\mathrm{C} 7$, whilst carotid atherosclerosis is a pan-vascular disease involving intra- and extra- cranial arteries. 3D CMR VWI images can cover the whole internal carotid artery within $7 \mathrm{~min}$. Assessment of lesions within a large FOV is useful for the selection of stent size and the formulation of surgical procedures [36].

There were several limitations in this study. Firstly, measurements of 3D CMR VWI were acquired using MPR. Therefore, discrepancy from mismatched slices of the carotid artery lesions between 3D CMR VWI and DSA may have compromised the agreement analysis. Secondly, 3D CMR VWI was still susceptible to motion and residual blood flow artifacts which occurred at certain anatomical locations, including carotid bifurcations and petrous and lacerum segments of ICAs, which can interfere with the correct assessment of the condition [22]. For example, 3D CMR VWI reader 1 misdiagnosed 6 artifacts as stenosis in our study. Thirdly, CMR angiography was not included in our imaging protocol. There were two main reasons for this design. First, our study primarily aimed to evaluate the diagnostic performance of 
3D CMR VWI in characterizing carotid disease by comparison with the clinical gold standard (DSA), but not to focus on the agreement analysis between 3D CMR VWI and MRA. Second, carotid CMR angiography acquired by time-of-flight (TOF) or contrastenhanced gradient recalled echo (GRE) typically has a much lower spatial resolution than 3D CMR VWI (isotropic $0.63 \mathrm{~mm}$ ).

\section{Conclusion}

3D CMR VWI can be used to accurately assess carotid atherosclerosis with high spatial resolution and large anatomical coverage in a single scan, suggesting that the imaging modality may serve in routine imaging workup with comprehensive information for both the vessel wall and lumen.

\section{Supplementary information}

Supplementary information accompanies this paper at https://doi.org/10. 1186/s12968-020-0604-x.

Additional file 1: Online Figure 1. Example images of image quality at different scale levels. $A$, image quality score $=4$; $B$, image quality score $=3 ;$, image quality score $=2 ; \mathrm{D}$, image quality score $=1$.

\section{Abbreviations}

AUC: Area under the curve; 3D: Three-dimensional; BB: Black blood; CCA: Common carotid artery; Cl: Confidence interval; CMR: Cardiovascular magnetic resonance; CSF: Cerebral spinal fluid; DSA: Digital subtraction angiography; FOV: Field-of-view; GRE: Gradient recalled echo; HDL: High density lipoprotein; ICA: Internal carotid artery; ICC: Intraclass correlation coefficient; IQ: Image quality; LDL: Low density lipoprotein; LP(a): Lipoprotein (a); MPR: Multiplanar reconstruction; MTHFR677C-

T: Methylenetetrahydrofolate reductase 677C-T; NPV: Negative predictive value; PPV: Positive predictive value; ROC: Receiver operator characteristics; SD: Standard deviation; SE: Standard error; SNR: Signal-to-noise ratio; SPACE: Sampling Perfection with Application-optimized Contrast using different flip angle Evolutions; T1w: T1 weighted; TE: Echo time; TOF: Time-offlight; TR: Repetition time; US: Ultrasound; WWI: Vessel wall imaging

\section{Acknowledgements}

We thank all of the patients for participating in this research.

\section{Authors' contributions}

ZW performed data acquisition, analysis, and interpretation and drafted the manuscript. WL and TZ made substantial contributions to data acquisition and analysis. ML was in charge of statistical analyses and data interpretation. ZF has made substantial contributions to the study design and data interpretation and helped to revise the manuscript critically for important intellectual content. DL has made a substantial contribution to the study design. WY has made substantial contributions to conception and design of the study. All authors read and approved the final manuscript.

\section{Funding}

This work was supported by grants from the Natural Science Foundation of Beijing $(17$ L20194, 7182046) and National Institutes of Health (NIH/NHLBI 1R01HL147355).

\section{Availability of data and materials}

The datasets used and/or analyzed during the current study are available from the corresponding author on reasonable request.

\section{Ethics approval and consent to participate}

The study design was approved by the Ethical Board of Beijing Anzhen Hospital. Written informed consent was obtained from all study participants.

\section{Consent for publication}

Written informed consent for the publication was obtained from all subjects.

\section{Competing interests}

The authors declare that they have no competing interests.

\section{Author details}

'Department of Radiology, Beijing Anzhen Hospital, Capital Medical University, No.2 Anzhen Road, Beijing 100029, China. ²Department of Radiology, Beijing Hospital of Traditional Chinese Medicine, Capital Medical University, No. 23, Back Road of Art Gallery, Beijing 100010, China. ${ }^{3}$ Department of Otolaryngology Head and Neck Surgery, Beijing Anzhen Hospital, Capital Medical University, No. 2 Anzhen Road, Beijing 100029, China. ${ }^{4}$ Department of Neurosurgery, Beijing Anzhen Hospital, Capital Medical University, No. 2 Anzhen Road, Beijing 100029, China. ${ }^{5}$ Biomedical Imaging Research Institute, Department of Biomedical Sciences, Cedars-Sinai Medical Center, 8700 Beverly Blvd., PACT 400, Los Angeles, CA 90048, USA. ${ }^{6}$ Department of Bioengineering, University of California, Los Angeles, CA, USA.

Received: 24 May 2019 Accepted: 5 February 2020

Published online: 05 March 2020

\section{References}

1. Barnett HJ, Gunton RW, Eliasziw M, et al. Causes and severity of ischemic stroke in patients with internal carotid artery stenosis. JAMA. 2000;283(11): 1429-36.

2. GBD 2016 Neurology Collaborators. Global, regional, and national burden of neurological disorders, 1990-2016: a systematic analysis for the Global Burden of Disease Study 2016. Lancet Neurol. 2019;18(5):459-80.

3. Fereydooni A, Gorecka J, Xu J, Schindler J, Dardik A. Carotid Endarterectomy and Carotid Artery Stenting for Patients With Crescendo Transient Ischemic Attacks: A Systematic Review. JAMA Surg. 2019;4(9). https://doi.org/10.1001/ jamasurg.2019.2952

4. Yamada K, Yoshimura S, Shirakawa M, et al. Asymptomatic moderate carotid artery stenosis with intraplaque hemorrhage: progression of degree of stenosis and new ischemic stroke. J Clin Neurosci. 2019;6(3):95-9.

5. Gogela SL, Gozal YM, Zhang B, et al. Severe carotid stenosis and delay of reperfusion in endovascular stroke treatment: an interventional Management of Stroke-III study. J Neurosurg. 2018;128(1):94-9.

6. Liem Ml, Kennedy F, Bonati LH, et al. Investigations of carotid stenosis to identify vulnerable atherosclerotic plaque and determine individual stroke risk. Circ J. 2017:81(9):1246-53.

7. North American Symptomatic Carotid Endarterectomy Trial. Methods, patient characteristics, and progress. Stroke. 1991;22(6):711-20.

8. Moore WS, Barnett HJ, Beebe HG, et al. Guidelines for carotid endarterectomy. A multidisciplinary consensus statement from the ad hoc committee, American Heart Association. Circulation. 1995;91(2):566-79.

9. Bettmann MA, Heeren T, Greenfield A, Goudey C. Adverse events with radiographic contrast agents: results of the SCVIR contrast agent registry. Radiology. 1997;203(3):611-20.

10. Yamauchi K, Enomoto Y, Otani K, Egashira Y, Iwama T. Prediction of hyperperfusion phenomenon after carotid artery stenting and carotid angioplasty using quantitative DSA with cerebral circulation time imaging. J Neurointerv Surg. 2018;10(6):576-9.

11. Saba L, Yuan C, Hatsukami TS, et al. Carotid Artery Wall Imaging: Perspective and Guidelines from the ASNR Vessel Wall Imaging Study Group and Expert Consensus Recommendations of the American Society of Neuroradiology. AJNR Am J Neuroradiol. 2018;39(2):E9-9E31.

12. Cai JM, Hatsukami TS, Ferguson MS, Small R, Polissar NL, Yuan C. Classification of human carotid atherosclerotic lesions with in vivo multicontrast magnetic resonance imaging. Circulation. 2002;106(11): 1368-73.

13. Cai J, Hatsukami TS, Ferguson MS, et al. In vivo quantitative measurement of intact fibrous cap and lipid-rich necrotic core size in atherosclerotic carotid plaque: comparison of high-resolution, contrast-enhanced magnetic resonance imaging and histology. Circulation. 2005:112(22):3437-44.

14. Saam T, Raya JG, Cyran CC, et al. High resolution carotid black-blood 3T MR with parallel imaging and dedicated 4-channel surface coils. J Cardiovasc Magn Reson. 2009;11:41. 
15. Yoshida K, Narumi O, Chin M, et al. Characterization of carotid atherosclerosis and detection of soft plaque with use of black-blood MR imaging. AJNR Am J Neuroradiol. 2008;29(5):868-74.

16. Qiao Y, Steinman DA, Qin Q, et al. Intracranial arterial wall imaging using three-dimensional high isotropic resolution black blood MRI at 3.0 tesla. J Magn Reson Imaging. 2011;34(1):22-30.

17. Li L, Chai JT, Biasiolli L, et al. Black-blood multicontrast imaging of carotid arteries with DANTE-prepared 2D and 3D MR imaging. Radiology. 2014; 273(2):560-9.

18. Li F, Yarnykh VL, Hatsukami TS, et al. Scan-rescan reproducibility of carotid atherosclerotic plaque morphology and tissue composition measurements using multicontrast MRI at 3T. J Magn Reson Imaging. 2010;31(1):168-76.

19. Lu M, Peng P, Cui Y, et al. Association of Progression of carotid Artery Wal volume and recurrent transient ischemic attack or stroke: a magnetic resonance imaging study. Stroke. 2018;49(3):614-20.

20. Zhao H, Wang J, Liu X, et al. Assessment of carotid artery atherosclerotic disease by using three-dimensional fast black-blood MR imaging: comparison with DSA. Radiology. 2015;274(2):508-16.

21. Qiao Y, Zeiler SR, Mirbagheri S, et al. Intracranial plaque enhancement in patients with cerebrovascular events on high-spatial-resolution MR images. Radiology. 2014;271(2):534-42.

22. Xie Y, Yang Q, Xie G, Pang J, Fan Z, Li D. Improved black-blood imaging using DANTE-SPACE for simultaneous carotid and intracranial vessel wal evaluation. Magn Reson Med. 2016;75(6):2286-94.

23. Fan Z, Yang Q, Deng Z, et al. Whole-brain intracranial vessel wall imaging at 3 tesla using cerebrospinal fluid-attenuated T1-weighted 3D turbo spin echo. Magn Reson Med. 2017;77(3):1142-50.

24. Yang Q, Deng Z, Bi X, et al. Whole-brain vessel wall MRI: a parameter tuneup solution to improve the scan efficiency of three-dimensional variable flip-angle turbo spin-echo. J Magn Reson Imaging. 2017:46(3):751-7.

25. Fan Z, Zhang Z, Chung YC, et al. Carotid arterial wall MRI at 3T using 3D variable-flip-angle turbo spin-echo (TSE) with flow-sensitive dephasing (FSD). J Magn Reson Imaging. 2010;31(3):645-54.

26. Fan Z, Zuehlsdorff S, Liu X, Li D. Prospective self-gating for swallowing motion: a feasibility study in carotid artery wall MRI using three-dimensional variable-flip-angle turbo spin-echo. Magn Reson Med. 2012;67(2):490-8.

27. Zhang N, Zhang F, Deng Z, et al. 3D whole-brain vessel wall cardiovascular magnetic resonance imaging: a study on the reliability in the quantification of intracranial vessel dimensions. J Cardiovasc Magn Reson. 2018;20(1):39.

28. Underhill HR, Yuan C, Terry JG, et al. Differences in carotid arterial morphology and composition between individuals with and without obstructive coronary artery disease: a cardiovascular magnetic resonance study. J Cardiovasc Magn Reson. 2008;10:31.

29. Sun B, Zhao H, Liu X, et al. Elevated hemoglobin A1C is associated with carotid plaque vulnerability: novel findings from magnetic resonance imaging study in hypertensive stroke patients. Sci Rep. 2016;6:33246.

30. Bouthillier A, van Loveren HR, Keller JT. Segments of the interna carotid artery: a new classification. Neurosurgery. 1996;38(3):425-32 discussion 432-433.

31. Haynes RB, Taylor DW, Sackett DL, Thorpe K, Ferguson GG, Barnett HJ. Prevention of functional impairment by endarterectomy for symptomatic high-grade carotid stenosis. North American symptomatic carotid Endarterectomy trial collaborators. JAMA. 1994;271(16):1256-9.

32. Curvo-Semedo L, Lambregts DM, Maas M, et al. Rectal cancer: assessment of complete response to preoperative combined radiation therapy with chemotherapy--conventional MR volumetry versus diffusion-weighted MR imaging. Radiology. 2011;260(3):734-43.

33. Glagov S, Weisenberg E, Zarins CK, Stankunavicius R, Kolettis GJ. Compensatory enlargement of human atherosclerotic coronary arteries. $\mathrm{N}$ Engl J Med. 1987;316(22):1371-5.

34. Liu T, Maurovich-Horvat P, Mayrhofer T, et al. Quantitative coronary plaque analysis predicts high-risk plaque morphology on coronary computed tomography angiography: results from the ROMICAT II trial. Int J CardiovasC Imaging. 2018;34(2):311-9.

35. Etesami M, Hoi Y, Steinman DA, et al. Comparison of carotid plaque ulcer detection using contrast-enhanced and time-of-flight MRA techniques. AJNR Am J Neuroradiol. 2013;34(1):177-84.

36. Hasan D, Zanaty M, Starke RM, et al. Feasibility, safety, and changes in systolic blood pressure associated with endovascular revascularization of symptomatic and chronically occluded cervical internal carotid artery using a newly suggested radiographic classification of chronically occluded cervical internal carotid artery: pilot study. J Neurosurg. 2018:1-10.

\section{Publisher's Note}

Springer Nature remains neutral with regard to jurisdictional claims in published maps and institutional affiliations.
Ready to submit your research? Choose BMC and benefit from:

- fast, convenient online submission

- thorough peer review by experienced researchers in your field

- rapid publication on acceptance

- support for research data, including large and complex data types

- gold Open Access which fosters wider collaboration and increased citations

- maximum visibility for your research: over $100 \mathrm{M}$ website views per year

At BMC, research is always in progress.

Learn more biomedcentral.com/submissions 